\title{
A Study of Different Type of H-Curvature Tensors in Sasakian Manifold
}

\author{
T.S. Chauhan \\ Associate Professor, Department of Maths, Bareilly College, Bareilly(U.P.), India
}

\begin{abstract}
Purpose of the present paper is to study of different H-Curvature tensors in Sasakian manifold. In section 1 is devoted for introduction. Section 2 deals to the study of H-Projective curvature tensor, H-Conformal curvature tensor, H-Conharmonic curvature tensor, H-Concircular tensor in Sasakian manifold.
\end{abstract}

Keywords: H-Projective curvature tensor, H-Conformal curvature tensor, H-Conharmonic curvature tensor, H-Concircular tensor

\section{Introduction}

An n-dimensional Sasakian manifold $\mathrm{M}^{\mathrm{n}}$ is an odd dimensional Riemannian space, which admits a unit killing vector field $\eta^{\lambda}$ satisfying:

(1.1) $\quad \eta_{\mathrm{k}, \mathrm{i}, \mathrm{j}}=\eta_{\mathrm{j}} \mathrm{g}_{\mathrm{ik}}-\eta_{\mathrm{k}} \mathrm{g}_{\mathrm{ij}}$

Wherein a comma (,) followed by index denotes the operation of covariant differentiation with regard to the fundamental tensor $\mathrm{g}_{\mathrm{ij}}$ of the Riemannian space.

(1.2) $\quad \mathrm{R}_{\mathrm{ijk}}^{\mathrm{h}}=\partial_{\mathrm{i}}\left\{\mathrm{j}_{\mathrm{k}}^{\mathrm{h}}\right\}-\partial_{\mathrm{j}}\left\{\mathrm{i} \mathrm{k}_{\mathrm{k}}^{\mathrm{h}}\right\}+\left\{\mathrm{i}_{1}^{\mathrm{h}}\right\}\left\{\mathrm{l}_{\mathrm{jk}}^{\mathrm{l}}\right\}-\left\{\mathrm{j}_{\mathrm{l}}^{\mathrm{h}}\right\}\left\{\begin{array}{l}\mathrm{l} \\ \mathrm{ik}\end{array}\right\}$

Whereas the Ricci tensor and the scalar curvature are respectively given by
(1.3) $\quad \mathrm{R}_{\mathrm{jk}}=\mathrm{R}_{\mathrm{ijk}}^{\mathrm{i}}$,
(1.4) $\mathrm{R}=\mathrm{R}_{\mathrm{jk}} \mathrm{g}$
and
(1.5) $\quad \partial_{\mathrm{i}}=\left(\partial / \partial \mathrm{x}^{\mathrm{i}}\right)$

A tensor $\mathrm{S}_{\mathrm{ij}}$ is defined as

(1.6) $\mathrm{S}_{\mathrm{ij}}=-\mathrm{F}_{\mathrm{i}}^{\mathrm{a}} \mathrm{R}_{\mathrm{aj}}$

then we have

(1.7) $\quad \mathrm{S}_{\mathrm{ij}}=-\mathrm{S}_{\mathrm{ji}}$

and

(1.8) $\quad \mathrm{F}_{\mathrm{i}}^{\mathrm{a}} \mathrm{S}_{\mathrm{aj}}=-\mathrm{S}_{\mathrm{ia}} \mathrm{F}_{\mathrm{j}}^{\mathrm{a}}$.

\section{H-Curvature Tensor}

H-Projective curvature tensor in the Sasakian manifold is defined as [5]:

(2.1) $\quad \mathrm{P}^{\mathrm{h}}{ }_{\mathrm{ijk}}=\mathrm{R}_{\mathrm{ijk}}^{\mathrm{h}}+\{1 /(\mathrm{n}+2)\}\left(\mathrm{R}_{\mathrm{ik}} \delta^{\mathrm{h}}{ }_{\mathrm{j}}-\mathrm{R}_{\mathrm{jk}} \delta^{\mathrm{h}}{ }_{\mathrm{i}}+\mathrm{S}_{\mathrm{ik}} \mathrm{F}_{\mathrm{j}}^{\mathrm{h}}\right.$ $-S_{j k} F_{i}^{h}+2 S_{i j} F_{k}^{h}$ ).

\section{Definition 2.1:}

A Sasakian manifold is called H-Projective Recurrent if it satisfies the following condition

(2.2) $\quad \nabla_{1} \mathrm{P}_{\mathrm{ijk}}^{\mathrm{h}}=\lambda_{\mathrm{l}} \mathrm{P}_{\mathrm{ijk}}^{\mathrm{h}}$.

Wherein $\lambda_{1}$ is H-Projective recurrent vector.

\section{Definition 2.2:}

A Sasakian manifold is said to be H-Projective symmetric if it satisfies the following condition

(2.3) $\quad \nabla_{1} P^{\mathrm{h}}{ }_{\mathrm{ijk}}=0$.

\section{$\underline{\text { Definition 2.3: }}$}

A Sasakian manifold is termed as H-Projectively flat if (2.4) $\mathrm{P}_{\mathrm{ijk}}^{\mathrm{h}}=0$.

In this regard, we have the following theorem:

\section{Theorem 2.1:}

If Sasakian manifold is H-projectively flat then the Ricci tensor holds the relation $\mathrm{R}_{\mathrm{ij}}=-\{2 /(\mathrm{n}+2)\}\left(\mathrm{S}_{\mathrm{in}} \mathrm{F}_{\mathrm{j}}^{\mathrm{n}}+\mathrm{F}\right.$ $\mathrm{S}_{\mathrm{ij}}$ ).

\section{Proof:}

Transvecting equation (2.1) by $\mathrm{g}_{\mathrm{hm}}$, we get (2.5) $\quad \mathrm{P}_{\mathrm{ijkm}}=\mathrm{R}_{\mathrm{ijkm}}+\{1 /(\mathrm{n}+2)\}\left(\mathrm{g}_{\mathrm{jm}} \mathrm{R}_{\mathrm{ik}}-\mathrm{g}_{\mathrm{im}} \mathrm{R}_{\mathrm{jk}}+\mathrm{S}_{\mathrm{ik}} \mathrm{F}_{\mathrm{jm}}\right.$ $\left.\mathrm{S}_{\mathrm{jk}} \mathrm{F}_{\mathrm{im}}+2 \mathrm{~S}_{\mathrm{ij}} \mathrm{F}_{\mathrm{km}}\right)$.

Transvecting equation (2.5) with $\mathrm{g}^{\mathrm{km}}$ and using equation (1.8), we get

(2.6) $\quad \mathrm{P}_{\mathrm{ij}}=\mathrm{R}_{\mathrm{ij}}+\{2 /(\mathrm{n}+2)\}\left(\mathrm{S}_{\mathrm{in}} \mathrm{F}^{\mathrm{n}}{ }_{\mathrm{j}}+\mathrm{F} \mathrm{S}_{\mathrm{ij}}\right)$.

If a Sasakian manifold is H-projectively flat then equation (2.6) becomes reduced in the form

$$
\mathrm{R}_{\mathrm{ij}}=-\{2 /(\mathrm{n}+2)\}\left(\mathrm{S}_{\mathrm{in}} \mathrm{F}_{\mathrm{j}}^{\mathrm{n}}+\mathrm{F} \mathrm{S}_{\mathrm{ij}}\right) \text {. }
$$
$\mathrm{H}$-Concircular tensor is given by

(2.8) $\quad \mathrm{C}_{\mathrm{ijk}}^{\mathrm{h}}=\mathrm{R}_{\mathrm{ijk}}^{\mathrm{h}}+\{\mathrm{R} / \mathrm{n}(\mathrm{n}+2)\}\left(\mathrm{g}_{\mathrm{ik}} \delta_{\mathrm{j}}^{\mathrm{h}}-\mathrm{g}_{\mathrm{jk}} \delta^{\mathrm{h}}{ }_{\mathrm{i}}+\mathrm{S}_{\mathrm{ik}} \mathrm{F}_{\mathrm{j}}^{\mathrm{h}}\right.$ $\mathrm{S}_{\mathrm{jk}} \mathrm{F}_{\mathrm{i}}^{\mathrm{h}}+2 \mathrm{~F}_{\mathrm{ij}} \mathrm{F}_{\mathrm{k}}^{\mathrm{h}}$ ).

\section{Definition 2.4:}

A Sasakian manifold is called Sasakian manifold with recurrent $\mathrm{H}$-Concircular curvature tensor, if it satisfies

(2.9) $\quad \nabla_{1} C^{\mathrm{h}}{ }_{\mathrm{ijk}}=\lambda_{\mathrm{l}} \mathrm{C}_{\mathrm{ijk}}^{\mathrm{h}}$

for some non-zero recurrence vector $\lambda_{1}$.

\section{Definition 2.5:}

A Sasakian manifold is said to be H-Concircular symmetric if it satisfies the following condition

(2.10) $\quad \nabla_{1} \mathrm{C}_{\mathrm{ijk}}^{\mathrm{h}}=0$.

\section{Definition 2.6:}

A Sasakian manifold is termed as $\mathrm{H}$-Concircular flat if (2.11) $\mathrm{C}^{\mathrm{h}}{ }_{\mathrm{ijk}}=0$.

$\mathrm{H}$-Conharmonic curvature tensor is given by 


\section{International Journal of Science and Research (IJSR) \\ ISSN (Online): 2319-7064}

Index Copernicus Value (2015): 78.96 | Impact Factor (2015): 6.391

(2.12) $\quad \mathrm{T}_{\mathrm{ijk}}^{\mathrm{h}}=\mathrm{R}_{\mathrm{ijk}}^{\mathrm{h}}+\{1 /(\mathrm{n}+4)\}\left(\mathrm{R}_{\mathrm{ik}} \delta^{\mathrm{h}}{ }_{\mathrm{j}}-\mathrm{R}_{\mathrm{jk}} \delta_{\mathrm{i}}^{\mathrm{h}}+\mathrm{g}_{\mathrm{ik}} \mathrm{R}_{\mathrm{j}}^{\mathrm{h}}-\mathrm{g}_{\mathrm{jk}}\right.$ $\left.\mathrm{R}_{\mathrm{i}}^{\mathrm{h}}+\mathrm{S}_{\mathrm{ik}} \mathrm{F}_{\mathrm{j}}^{\mathrm{h}}-\mathrm{S}_{\mathrm{jk}} \mathrm{F}_{\mathrm{i}}^{\mathrm{h}}+\mathrm{F}_{\mathrm{ik}} \mathrm{S}_{\mathrm{j}}^{\mathrm{h}}-\mathrm{F}_{\mathrm{jk}} \mathrm{S}_{\mathrm{i}}^{\mathrm{h}}+2 \mathrm{~S}_{\mathrm{ij}} \mathrm{F}_{\mathrm{k}}^{\mathrm{h}}+2 \mathrm{~F}_{\mathrm{ij}} \mathrm{S}_{\mathrm{k}}^{\mathrm{h}}\right)$

\section{Definition 2.7:}

A Sasakian space satisfying the following condition (2.13) $\quad \nabla_{1} \mathrm{~T}^{\mathrm{h}}{ }_{\mathrm{ijk}}=\lambda_{1} \mathrm{~T}^{\mathrm{h}}{ }_{\mathrm{ijk}}$

for some non-zero recurrence vector $\lambda_{1}$, will be called a Sasakian manifold with recurrent $\mathrm{H}$-Conharmonic curvature tensor.

\section{Definition 2.8:}

A Sasakian manifold is said to be H-Conharmonic symmetric if it satisfies the following condition
(2.14)
$\nabla_{1} \mathrm{~T}^{\mathrm{h}}{ }_{\mathrm{ijk}}=0$.

\section{Definition 2.9:}

A Sasakian manifold is termed as H-Conharmonic flat if (2.15) $\mathrm{T}_{\mathrm{ijk}}^{\mathrm{h}}=0$.

$\mathrm{H}$-Conformal curvature tensor in the Sasakian manifold is given by

(2.16) $\quad \mathrm{B}_{\mathrm{ijk}}^{\mathrm{h}}=\mathrm{R}_{\mathrm{ijk}}^{\mathrm{h}}+\{1 /(\mathrm{n}+4)\}\left(\mathrm{R}_{\mathrm{ik}} \delta^{\mathrm{h}}{ }_{\mathrm{j}}-\mathrm{R}_{\mathrm{jk}} \delta_{\mathrm{i}}^{\mathrm{h}}+\mathrm{g}_{\mathrm{ik}} \mathrm{R}_{\mathrm{j}}^{\mathrm{h}}\right.$ $\left.-g_{j k} R_{i}^{h}+S_{i k} F_{j}^{h}-S_{j k} F_{i}^{h}+F_{i k} S_{j}{ }_{j} F_{j k} S_{i}^{h}+2 S_{i j} F_{k}^{h}+2 F_{i j} S_{k}^{h}\right)$

- $\{\mathrm{R} /(\mathrm{n}+2)(\mathrm{n}+4)\}\left(\mathrm{g}_{\mathrm{ik}} \delta^{\mathrm{h}}{ }_{\mathrm{j}}-\mathrm{g}_{\mathrm{jk}} \delta^{\mathrm{h}}{ }_{\mathrm{i}}+\mathrm{F}_{\mathrm{ik}} \mathrm{F}_{\mathrm{j}}^{\mathrm{h}}-\mathrm{F}_{\mathrm{jk}} \mathrm{F}_{\mathrm{i}}+2 \mathrm{~F}_{\mathrm{ij}} \mathrm{F}_{\mathrm{k}}^{\mathrm{h}}\right)$.

\section{Definition 2.10:}

A Sasakian space satisfying the relation

$$
\text { (2.17) } \quad \nabla_{1} \mathrm{~B}^{\mathrm{h}}{ }_{\mathrm{ijk}}=\lambda_{\mathrm{l}} \mathrm{B}^{\mathrm{h}}{ }_{\mathrm{ijk}}
$$

is termed as Sasakian space with recurrent $\mathrm{H}$-Conformal curvature tensor.

\section{Definition 2.11:}

A Sasakian manifold is said to be $\mathrm{H}$-Conformal symmetric if it satisfies the following condition

$$
\text { (2.18) } \quad \nabla_{1} \mathrm{~B}_{\mathrm{ijk}}^{\mathrm{h}}=0 \text {. }
$$

\section{Definition 2.12:}

A Sasakian manifold is termed as H-Conformal flat if (2.19) $\mathrm{B}^{\mathrm{h}}{ }_{\mathrm{ijk}}=0$.

From equations (2.12) and (2.16), we get (2.20) $\quad \mathrm{B}^{\mathrm{h}}{ }_{\mathrm{ijk}}=\mathrm{T}^{\mathrm{h}}{ }_{\mathrm{ijk}}-\{\mathrm{R} /(\mathrm{n}+2)(\mathrm{n}+4)\}\left(\mathrm{g}_{\mathrm{ik}} \delta^{\mathrm{h}}{ }_{\mathrm{j}}-\mathrm{g}_{\mathrm{jk}} \delta^{\mathrm{h}}{ }_{\mathrm{i}}+\mathrm{F}_{\mathrm{ik}} \mathrm{F}^{\mathrm{h}}{ }_{\mathrm{j}}\right.$ $-\mathrm{F}_{\mathrm{jk}} \mathrm{F}_{\mathrm{i}}^{\mathrm{h}}+2 \mathrm{~F}_{\mathrm{ij}} \mathrm{F}_{\mathrm{k}}^{\mathrm{h}}$ ).

By virtue of equations (2.8) and (2.20), we obtain (2.21) $\quad \mathrm{B}_{\mathrm{ijk}}^{\mathrm{h}}=\mathrm{T}_{\mathrm{ijk}}^{\mathrm{h}}+\{\mathrm{n} /(\mathrm{n}+4)\}\left(\mathrm{R}_{\mathrm{ijk}}^{\mathrm{h}}=\mathrm{C}_{\mathrm{ijk}}^{\mathrm{h}}\right)$,

In this regard, we have the following theorems:

\section{Theorem 2.2:}

In a Sasakian manifold is $\mathrm{H}$-projective curvature tensor, $\mathrm{H}$ concircular tensor and $\mathrm{H}$-conharmonic curvature tensor are flat then $\mathrm{H}$-conformal curvature tensor is also flat.

\section{Proof:}

By virtue of equations (2.4), (2.11), (2.15), (2.19) and (2.21).

\section{Theorem 2.3:}

In a Sasakian manifold is $\mathrm{H}$-projective curvature tensor, $\mathrm{H}$ concircular tensor and $\mathrm{H}$ - conformal curvature tensor are flat then $\mathrm{H}$-conharmonic curvature tensor is also flat.

\section{Proof:}

By virtue of equations (2.4), (2.11), (2.15), (2.19) and (2.21).

\section{Theorem 2.4:}

In a Sasakian manifold is $\mathrm{H}$-projective curvature tensor, $\mathrm{H}$ conformal tensor and $\mathrm{H}$-conharmonic curvature tensor are flat then $\mathrm{H}$-concircular curvature tensor is also flat.

\section{Proof:}

By virtue of equations (2.4), (2.11), (2.15), (2.19) and (2.21).

\section{Theorem 2.5:}

In a Sasakian manifold is $\mathrm{H}$-conformal curvature tensor, $\mathrm{H}$ concircular tensor and $\mathrm{H}$-conharmonic curvature tensor are flat then $\mathrm{H}$ - projective curvature tensor is also flat.

\section{Proof:}

By virtue of equations (2.4), (2.11), (2.15), (2.19) and (2.21).

\section{References}

[1] Amur Krishna and S.S. Pujar : Conformalily and isometry to spheres of Riemannian manifolds to spheres II, Jon. Differential Geometry, 13(2), p.(243-350), (1978).

[2] K. Yano : Groups of transformations in generalized spaces, Akademeia Press, Tokyo, (1949).

[3] K. Yano : Integral formulas in Riemannian Geometry, Marcel Dekkar, New York, (1970).

[4] K. Yano : On Sasakian manifolds with constant scalar curvature admitting a conformal transformation group, Proc. Nat. Acad. Sci. U.S.A., p.(314-319), 62(1969).

[5] K. Yano : Sasakian manifolds admitting a conformal transformation group, Proc. Nat. Acad. Sci. U.S.A., p.(472-476), 55(1966).

[6] S.M. Bhati and S.S. Pujar : On Sasakian manifolds with constant scalar curvature admitting aconformal transformation group, Acta Ciencia Indica,Vol.XXXII M, No.1, 161(2006).

\section{Author Profile}

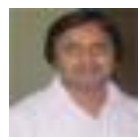

T.S. Chauhan (Tarkeshwar Singh Chauhan) received Ph.D. and D.Sc. degrees in Mathematics from M.J.P.R.U., Bareilly in 1992 and 2008 respectively. He has been working in Maths deptt., Bareilly College, Bareilly since 1990 and now he is an Associate Professor. Under his guidance nearly 25 candidates have been awarded Ph.D. degree. Several papers and books are published in different branches in different publications under him. 